\title{
BMJ Global Health Insulin prices, availability and affordability in 13 low-income and middle-income countries
}

\author{
Margaret Ewen, ${ }^{1}$ Huibert-Jan Joosse, ${ }^{2}$ David Beran, ${ }^{\circ}$ Richard Laing ${ }^{4}$
}

To cite: Ewen M, Joosse H-J,

Beran D, et al. Insulin

prices, availability and affordability in 13 lowincome and middle-income countries. BMJ Global Health 2019;4:e001410. doi:10.1136/ bmjgh-2019-001410

Handling editor Seye Abimbola

- Additional material is published online only. To view please visit the journal online (http://dx.doi.org/10. 1136bmjgh-2019-001410)

Received 11 January 2019 Revised 15 April 2019 Accepted 19 April 2019
D Check for updates

C) Author(s) (or their employer(s)) 2019. Re-use permitted under CC BY-NC. No commercial re-use. See rights and permissions. Published by BMJ.

${ }^{1}$ Health Action International, Amsterdam, The Netherlands ${ }^{2}$ Utrecht, The Netherlands ${ }^{3}$ Division of Tropical and Humanitarian Medicine, University of Geneva and Geneva University Hospitals, Geneva, Switzerland ${ }^{4}$ Center for Global Health, Boston University School of Public Health, Boston, Massachusetts, USA

Correspondence to Dr Margaret Ewen; marg@haiweb.org

\section{ABSTRACT}

Introduction Globally, one in two people needing insulin lack access. High prices and poor availability are thought to be key contributors to poor insulin access. However, few studies have assessed the availability, price and affordability of different insulin types in low-income and middle-income countries in a systematic way.

Methods In 2016, 15 insulin price and availability surveys were undertaken (using an adaptation of the $\mathrm{WHO}$ / Health Action International medicine price and availability measurement methodology) in Brazil, China (Hubei and Shaanxi Provinces), Ethiopia, Ghana, India (Haryana and Madhya Pradesh States), Indonesia, Jordan, Kenya, Kyrgyzstan, Mali, Pakistan, Russia (Kazan Province) and Uganda. Data were collected in three sectors (public, private pharmacies and private hospitals/clinics) in three regions per survey. Insulin prices were standardised to $10 \mathrm{~mL} 100 \mathrm{IU} / \mathrm{mL}$ in US dollars (\$). Data were also collected for four comparator medicines.

Results Mean availability was higher for human (55\%-80\%) versus analogue insulins $(55 \%-63 \%)$, but only short-acting human insulin reached $80 \%$ availability (public sector). Median government procurement prices

\section{Key questions}

\section{What is already known?}

- Despite being discovered nearly 100 years ago, one in every two people globally needing insulin lack access to this life-saving medicine.

- The global insulin market is dominated by only three companies, but it is estimated that increased competition with biosimilar insulin could lead to large price reductions.

What are the new findings?

- In low-income and middle-income countries, insulin availability in outlets is poor, and treatment is unaffordable for those on low wages having to pay out of pocket.

- Biosimilar insulins are mostly (but not always) cheaper than originator brands, and analogue insulins, in particular, are far higher priced than estimated costs of production including profit.

What do the new findings imply?

- High insulin prices and poor availability need to be addressed through national and global actions. were $\$ 5$ (human insulins) and \$33 (long-acting analogues). In all three sectors, median patient prices were $\$ 9$ for human insulins. Median patient prices for analogues varied between the public sector (\$34) and the two private sectors (\$44). Vials were cheaper than pens and cartridges. Biosimilars, when available, were mostly cheaper than originators. A low-income person had to work 4 and 7 days to buy $10 \mathrm{~mL}$ human and analogue insulin, respectively. For isophane human insulin, only three countries meet the WHO target of $80 \%$ availability of affordable essential medicines for non-communicable diseases in any sector. Conclusion Improving insulin availability and affordability needs to be addressed through national and global actions, including prioritising the supply of more affordable human insulin, increasing competition through the use of lower priced quality-assured biosimilars, negotiating lower prices from manufacturers and improving distribution systems.

\section{BACKGROUND}

By 2045, an estimated 629 million people will have diabetes. This constitutes a $48 \%$ increase over the 2017-2045 period. ${ }^{1}$ Currently, one in two people needing insulin lack access to this life-saving medicine. To address this, in 2013, the WHO published its Global Action Plan for the Prevention and Control of Non-communicable Diseases 2013-2020 (GAP). ${ }^{2}$ One of GAP's objectives is to strengthen health systems, which includes improving access to affordable medicines to treat non-communicable diseases (NCDs). The GAP includes a voluntary target of $80 \%$ availability of affordable essential medicines, including generics, required to treat major NCDs in both public and private facilities by 2025 . Data have shown that few essential medicines for diabetes have met the GAP target. ${ }^{3}$

In the public sector in low-income, lower middle income and upper middle income countries $17 \%, 21 \%$ and $45 \%$, respectively, of diabetes medicines were both available $(80 \%$ or greater availability in outlets) and affordable (requiring no more than 1 day's wages of the lowest paid unskilled government worker 
to purchase 30 days' treatment). In the private sector, it was $28 \%, 23 \%$ and $32 \% .^{3}$

Insulin is a life-saving medicine for people with type 1 diabetes and is used to manage an increasing number of people with type 2 diabetes. Serious complications result from suboptimal treatment, including blindness, amputations and premature death. Therefore, access to insulin is vital.

Studies in 2003-2009 by the International Insulin Foundation in seven low-income and middle-income countries (LMICs) found a variety of barriers to insulin access, including high prices. ${ }^{4-13}$ Availability was also suboptimal, for example, in Mali and Mozambique, insulin was present in only $20 \%$ of public sector outlets. In 2010, a snapshot survey by Health Action International (HAI) of 60 countries found patient prices of regular insulin were highly variable across the world. ${ }^{14}$ A snapshot 2015 survey (43 countries) showed insulin patient prices were generally high, and treatment was unaffordable for those on low incomes. ${ }^{15}$ Human insulins were lower priced than analogues in public sector outlets (median US $\$ 7.64$ vs $\$ 45.03$ for $10 \mathrm{~mL} 100 \mathrm{IU} / \mathrm{mL}$ ) and private pharmacies (median US $\$ 16.65$ vs $\$ 39.35$ ), with prices highly variable between countries. Insulin vials were cheaper than insulin cartridges and prefilled pens for all human insulins, but not all analogues. On average, 2.5-9.5 days' wages were needed, by those on a low wage, to buy $10 \mathrm{~mL}$ depending on the insulin type and sector. Government procurement prices were, on average, US\$5.99 for human insulin and US $\$ 34.20$ for analogues. ${ }^{15}$ A recently published study showed insulin availability in pharmacies was poor in LMICs, and $63 \%$ of households in low-income countries could not afford insulin. ${ }^{16}$

This article reports the findings from a comprehensive survey undertaken in 2016 as part of the Addressing the Challenge and Constraints of Insulin Sources and Supply (ACCISS) Study on the availability, price and affordability of insulin, and four comparator medicines, in the public and private sectors of 13 LMICs.

\section{METHODS}

\section{Sampling}

Fifteen surveys were undertaken in 13 countries: Brazil, China (Hubei and Shaanxi provinces), Ethiopia, Ghana, India (Haryana and Madhya Pradesh states), Indonesia, Jordan, Kenya, Kyrgyzstan, Mali, Pakistan, Russia (Kazan province) and Uganda. Three sectors were surveyed: public hospitals (outpatients dispensary), private retail pharmacies and private hospitals/clinics. Data were collected in three areas per survey: including the capital or major urban city, the province closest to this city with the largest provincial hospital and the district closest to the province with the largest district hospital. In each area, the largest public hospital and private hospital/ clinic were sampled, plus five private retail pharmacies (a large pharmacy and four randomly chosen from those within $5 \mathrm{~km}$ of the public hospital). In some countries, there were no private hospitals/clinics in the district. See online supplementary appendix table 1 for the number of outlets sampled per survey.

The latest government procurement (tender) prices were collected from the national procurement office or central medical stores. In Brazil, India Madhya Pradesh, Pakistan and Russia Kazan, where medicine procurement is decentralised, procurement prices were collected in the public hospitals surveyed.

\section{Medicines}

Patient price and availability data were collected for all insulins found in the outlets and for four comparator medicines. Two comparators are commonly used to treat type 2 diabetes: metformin $500 \mathrm{mg}$ tablets/capsules and gliclazide $80 \mathrm{mg}$ tablet/capsules. Enalapril $10 \mathrm{mg}$ tablets/ capsules (used to treat hypertension) and ceftriaxone 1 $\mathrm{g}$ vials (the most commonly used injectable antibiotic in LMICs) were also surveyed. In Brazil and Kyrgyzstan, gliclazide was not surveyed as no products in the specified strength and dosage form have marketing authorisation.

For the comparators, prices were collected on the highest and lowest unit prices in the outlet (irrespective of whether they were originator brands or generics). All data were entered into customised Excel spreadsheets.

\section{Data collection, entry and quality assurance}

In each country, the Ministry of Health endorsed the survey. The outlets gave verbal consent to data collection.

National investigators experienced in conducting medicine price surveys (including using the WHO/HAI methodology ${ }^{17}$ ) trained their survey personnel. The authors reviewed and analysed the data. Insulin types, brands, volumes of insulin per presentation and so on were checked against the manufacturer's website. Where a product list was not provided, websites of major distributors were used. Brands of comparator medicines were also checked to confirm the active ingredient, strength and dosage form.

\section{Patient and public involvement}

No patients or the general public were involved in the survey.

\section{Data analysis}

The insulins were categorised by time of action that is, short-acting and intermediate-acting human insulin, mixed human insulin, rapid-acting and long-acting analogue insulin, mixed analogue insulin and animal insulin (see online supplementary appendix table 2).

Availability was assessed as the percentage of outlets that stocked the medicine on the day of data collection. In the public sector, the level of health facility (primary, secondary or tertiary-level hospitals/health centres) at which the medicine is legally permitted to be dispensed, and hence should be available, was considered. Whether the medicine was on the National Essential Medicines List (NEML) was also considered. Mean availability per sector of each insulin category, plus originators versus 


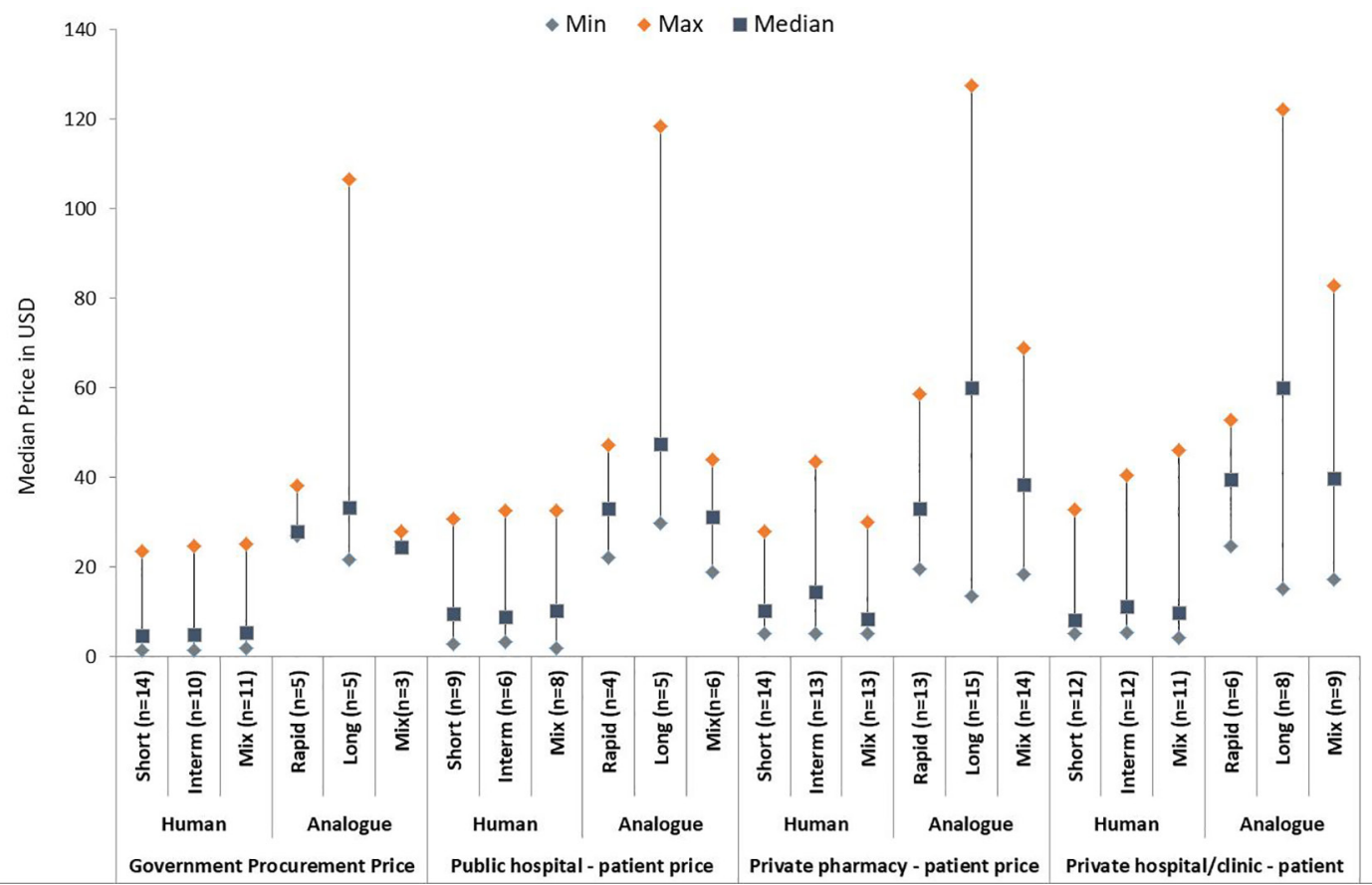

Figure 1 Median prices of insulin by category and sector, $10 \mathrm{~mL} 100 \mathrm{IU} / \mathrm{mL}$ in US dollars (any presentation).

biosimilars, and the comparators was assessed across the surveys.

Insulin prices were standardised to $10 \mathrm{~mL}$ of $100 \mathrm{IU} /$ $\mathrm{mL}$. Both insulin and comparator prices were converted to US dollars $(\$)$ using the exchange rate of the first day of data collection according to OANDA (www.oanda. com/currency/converter).

For each survey, median prices for each insulin category, type and presentation were assessed per outlet and per sector. Insulin prices were also compared between the originators (manufactured by Novo Nordisk A/S (Novo Nordisk), Eli Lilly and Company (Lilly), Sanofi S.A. (Sanofi)) and independent biosimilar manufacturers (including biosimilars made by the originator companies). For each comparator, median prices were assessed for the highest and lowest price. In this article, only availability and affordability data are reported for comparators.

Affordability was based on median treatment prices and expressed as the number of days the lowest paid unskilled government worker has to work to purchase 10 $\mathrm{mL}$ of insulin (approximately 30 days' supply), 90 tablets metformin, 30 tablets gliclazide, 30 tablets enalapril and 1 vial of ceftriaxone. The GAP target did not define affordability, so the WHO/HAI threshold of not more than 1 day's wage of the lowest paid unskilled government worker to purchase 30 days' supply was used.

\section{FINDINGS}

\section{Government procurement prices}

Government procurement prices were obtained in all countries except Kenya. For six surveys, insulin was procured from only one manufacturer, in three surveys from two manufacturers, for four surveys from three manufacturers and in one from six manufacturers.

Median procurement prices of human insulins across the countries (any presentation) were similar, ranging from $\$ 4.56$ (short acting) to $\$ 5.30$ (mixed human) (figure 1 and online supplementary appendix table 3). Large price variations were seen across the countries. For example, short-acting and intermediate-acting human insulins ranged from $\$ 1.45$ (Ethiopia) to approximately \$24 (Hubei, China).

Six countries had at least one analogue on their NEML (Russia, Kyrgyzstan, Indonesia, China, Jordan and Ghana). Of these, all but Ghana had purchased analogues. Long-acting analogues had the highest median price (\$33.36) and the largest price variation (\$21.56 (Indonesia) to \$106.52 (Hubei, China)).

In most countries, there were large price differences between human and analogue insulins. The Kyrgyzstan government, for example, paid \$3-\$4 for human insulins and \$24-\$32 for analogues. By contrast, in Indonesia, the price differential was smaller (human insulin \$15-\$19 and analogues \$22-\$28). The highest prices were found in the two Chinese provinces; median prices of human insulins were approximately $\$ 24$, and rapid-acting and long-acting analogues were approximately $\$ 38$ and $\$ 100$, respectively.

For short-acting and mixed human insulins, median prices of originator brands $(\$ 5.90)$ were higher than biosimilars $(\$ 3.55)$, whereas they were similar for intermediate-acting human insulin (\$5.90 vs \$5.79). Only the Shaanxi provincial government in China purchased biosimilar analogues. For matched pairs of human insulins (same insulin, strength and presentation) assessed 
Table 1 Paired government procurement prices, originator and biosimilar insulins, 1000 IU in US dollars

\begin{tabular}{|c|c|c|c|c|}
\hline Survey & $\begin{array}{l}\text { Human insulin, strength, } \\
\text { presentation }\end{array}$ & Originator(s) & Biosimilar(s) & $\begin{array}{l}\text { Difference biosimilar } \\
\text { to originator }\end{array}$ \\
\hline Pakistan & $\begin{array}{l}\text { Regular, isophane and mixed 30/70, } \\
100 \mathrm{IU} / \mathrm{mL} \text { vial }\end{array}$ & $\$ 2.38 \mathrm{~N}$ & $\$ 2.00 \mathrm{G}$ & $16 \% \downarrow$ \\
\hline Kazan, Russia & Regular 100 IU/mL vial & $\$ 6.07 \mathrm{~N}$ & $\$ 6.65 P$ & $10 \% \uparrow$ \\
\hline \multirow[t]{4}{*}{ Shaanxi, China } & Mixed 30/70 $40 \mathrm{IU} / \mathrm{mL}$ vial & $\$ 21.26 \mathrm{~N}$ & $\$ 18.90 \mathrm{D}$ & $11 \% \downarrow$ \\
\hline & Isophane $100 \mathrm{IU} / \mathrm{mL}$ cartridge & $\$ 28.35 \mathrm{~N}$ & \$24.23 U; \$25.21 D & $11 \%-14 \% \downarrow$ \\
\hline & Mixed 30/70 $100 \mathrm{IU} / \mathrm{mL}$ cartridge & $\$ 28.35 \mathrm{~N}$ & \$25.21 U; \$25.21 D & $11 \% \downarrow$ \\
\hline & Mixed 50/50 $100 \mathrm{IU} / \mathrm{mL}$ cartridge & $\$ 28.35 \mathrm{~N}$ & \$25.05 U; \$26.96 D & $5 \%-12 \% \downarrow$ \\
\hline \multirow[t]{7}{*}{ Hubei, China } & Regular $40 \mathrm{IU} / \mathrm{mL}$ vial & $\$ 20.70 \mathrm{~N}$ & $\$ 17.71 \mathrm{D}$ & $14 \% \downarrow$ \\
\hline & Isophane $40 \mathrm{IU} / \mathrm{mL}$ vial & $\$ 20.76 \mathrm{~N}$ & $\$ 17.71 \mathrm{D}$ & $14 \% \downarrow$ \\
\hline & Mixed 30/70 $40 \mathrm{IU} / \mathrm{mL}$ vial & $\$ 21.39 \mathrm{~N}$ & $\$ 17.94 \mathrm{D}$ & $16 \% \downarrow$ \\
\hline & Regular $100 \mathrm{IU} / \mathrm{mL}$ cartridge & $\$ 26.51 \mathrm{~L} ; \$ 26.53 \mathrm{~N}$ & $\begin{array}{l}\$ 23.18 \mathrm{Li} ; \$ 23.62 \mathrm{D} ; \\
\$ 27.41 \mathrm{Bt}\end{array}$ & $3 \% \uparrow-13 \% \downarrow$ \\
\hline & Isophane $100 \mathrm{IU} / \mathrm{mL}$ cartridge & $\$ 26.20 \mathrm{~L} ; \$ 27.47 \mathrm{~N}$ & $\begin{array}{l}\$ 23.35 \mathrm{Li} ; \$ 24.24 \mathrm{D} ; \\
\$ 28.11 \mathrm{Bt}\end{array}$ & $7 \% \uparrow-15 \% \downarrow$ \\
\hline & Mixed 30/70 $100 \mathrm{lU} / \mathrm{mL}$ cartridge & $\$ 26.44 \mathrm{~L} ; \$ 26.66 \mathrm{~N}$ & $\begin{array}{l}\$ 22.56 \mathrm{Li} ; \$ 23.80 \mathrm{D} ; \\
\$ 27.84 \mathrm{Bt}\end{array}$ & $5 \% \uparrow-15 \% \downarrow$ \\
\hline & Mixed 50/50 $100 \mathrm{lU} / \mathrm{mL}$ cartridge & $\$ 27.78 \mathrm{~N}$ & \$22.09 Li; \$26.23 D & $6 \%-20 \% \downarrow$ \\
\hline
\end{tabular}

Originator companies: L, Lilly; N, Novo Nordisk.

Biosimilar companies: Bt, Bioton; D, Tonghua Dongbao; G, Getz; Li, Lianbang; P, Pharmstandard; U, United.

for Pakistan, Russia Kazan and the two Chinese provinces, biosimilars were no more than $20 \%$ lower priced than originators (table 1). In a few cases, the biosimilar was higher priced, for example, $10 \%$ higher in Russia Kazan (regular insulin in vials) and 3\%, 5\% and $7 \%$ in Hubei, China, for regular, mixed $30 / 70$ and isophane cartridges, respectively.

Only Hubei, China, was purchasing animal (porcine) insulin. Prices were $\$ 9.20, \$ 10.12$ and $\$ 13.29$ for shortacting, intermediate-acting and mixed porcine insulins, respectively. These were lower than median prices of the human insulins procured.

\section{Availability in outlets}

In the public sector, mean availability of human insulins was $55 \%-80 \%$ depending on the country (see online supplementary appendix figure 1). Analogue availability was $55 \%-63 \%$ in the six countries with them on their NEML plus Kenya (found but not in the NEML). In private pharmacies, availability was lower at $45 \%-53 \%$ (human) versus $27 \%-36 \%$ (analogue), and in private hospitals, it was $54 \%-71 \%$ (human) versus $35 \%-51 \%$ (analogue).

While Pakistan had good availability of the three types of human insulin in all three sectors $(95 \%-100 \%)$, this was not the case in other countries. For example, in Haryana, India, where insulin is free in the public sector, no human insulin was found in the provincial and district public hospitals, and only short-acting insulin was in stock in the teaching hospital in the state capital. Availability was higher in private pharmacies and private hospitals, where people have to pay out of pocket.

Mean availability of biosimilars was lower than originators in the public $(17 \%$ versus $51 \%)$, private pharmacy $(8 \%$ versus $40 \%)$ and private hospital (10\% vs $52 \%)$ sectors. Biosimilars of human insulins had higher availability (11\%-36\% depending on type and sector) than analogues $(0 \%-17 \%)$. Most analogue biosimilars were glargine (made by Biocon, Gan\&Lee, Getz and Lilly). In the public sector, the availability of biosimilars exceeded that of originators only for human insulins and only in a few countries, that is, India Haryana (regular), Ethiopia and Pakistan (regular and isophane), Uganda (regular, isophane and mixed human insulin) and India Madhya Pradesh (regular and mixed human insulin). There was only one case in private pharmacies (in Uganda for glargine) and one case in private hospitals (Ethiopia, mixed human insulin).

The mean availability of metformin and ceftriaxone was similar to, or higher than, human and analogue insulin availability in all sectors. A mixed picture was seen for gliclazide and enalapril, depending on the sector and insulin category.

\section{Patient prices}

Overall

Insulin was provided free of charge in the public sector in India, Kyrgyzstan, Pakistan, Uganda, Russia (registered users only from public hospitals) and Brazil. Where people pay out of pocket, median prices were $\$ 9.36$ for 
human insulin versus $\$ 29.39$ for analogues in the public sector, $\$ 9.65$ (human insulin) versus $\$ 43.81$ (analogues) in private pharmacies and $\$ 9.02$ (human insulin) versus $\$ 45.26$ (analogues) in private hospitals/clinics.

\section{By insulin category and country}

In the public sector, median patient prices ranged from $\$ 8.78$ to $\$ 10.15$ for human insulin and $\$ 31.27-\$ 47.46$ for analogues, depending on the type (figure 1). In private pharmacies and private hospitals/clinics, prices ranged from $\$ 8.36$ to $\$ 14.52$ and from $\$ 8.07$ to $\$ 11.11$, respectively, for human insulins, and $\$ 33.00-\$ 60.05$ and \$39.44-\$59.96, respectively, for analogues.

The largest price variations were seen for long-acting analogues. In private pharmacies, which had the greatest number of price points, glargine ranged from $\$ 13.42$ in Madhya Pradesh, India, to $\$ 131.27$ in Brazil (median $\$ 59.94)$. Prices for detemir ranged from $\$ 27.22$ in Kazan, Russia, to $\$ 116.36$ in Shaanxi, China (median $\$ 59.70$ ). In private pharmacies, degludec was only found in Brazil (\$117.57).

\section{By presentation}

In private pharmacies (greatest number of data points), median prices of vials of short-acting, intermediate-acting and rapid-acting insulin, and mixed human, were lower than insulin in pens and cartridges (see online supplementary appendix figure 2). For example, mixed human insulin in vials, pens and cartridges had median prices of $\$ 6.76, \$ 18.16$ and $\$ 14.42$, respectively. For long-acting analogues, vials were higher priced; for mixed analogues, prices were similar across presentations. Note: this was not a paired analysis, and there were limited data for some presentations and insulin categories.

\section{Originators versus biosimilars}

Of the 49 matched pairs of originators and biosimilars by presentation, biosimilars had lower median prices in 36 cases (18 vials and 18 cartridges), were identical in three (all vials) and had higher prices in 10 cases (6 vials and 4 cartridges) (table 2). Matched data for pens was available for only one outlet (Indonesian private pharmacy) where the biosimilar was $12 \%$ cheaper.

In the public sector, median biosimilar prices were all cheaper (by $7 \%-26 \%$ for vials; $2 \%-25 \%$ for cartridges) than originators except for one case in Ethiopia. In private pharmacies, biosimilars were cheaper (by $3 \%-45 \%$ vials; $11 \%-26 \%$ cartridges) than originators in $16 / 26$ cases, the same price in $2 / 26$ cases and higher priced in $8 / 26$ cases (by $1 \%-26 \%$ vials; $1 \%-83 \%$ cartridges). In private hospitals, biosimilars were all cheaper (by 13\%-40\% vials; $4 \%-38 \%$ cartridges) than originators except for two cases in Madhya Pradesh.

\section{Affordability}

Based on median prices, the lowest paid unskilled government worker has to work 3.5-3.9 days (human) versus 6.1-7.9 days (analogue) to purchase $10 \mathrm{~mL} 100 \mathrm{IU} /$ $\mathrm{mL}$ of insulin in the public sector, depending on type.
In private pharmacies, it was 2.2-4.3 (human) versus 6.6-15.6 (analogues) days' wages. In private hospitals, 3.7-5.0 (human) versus 6.7-14.3 (analogues) days' wages were needed (table 3). Long-acting analogues were least affordable, requiring 7.9, 15.6 and 14.3 days' wages in the public sector, private pharmacies and private hospitals, respectively.

Insulin was less affordable than the four comparators in all sectors. For example, a month's supply of metformin (first-line treatment for type 2 diabetes), purchased in the public sector, requires a median of 1.3 (lowest priced) to 2.4 (highest priced) days' wages, whereas insulin requires 3.5 to 7.9 days' wages depending on the type of insulin. Gliclazide, also used in the management of type 2 diabetes, was also more affordable than insulin, even for highest priced products.

Figure 2 shows the availability and affordability of isophane (intermediate acting) human insulin by country and sector. Only the public sector in Brazil, Kyrgyzstan and Pakistan (where insulin is free of charge) and private hospitals in Brazil achieved the GAP target, although a few other countries came close. Glargine (long-acting analogue) did not achieve the target in any country/ sector (see online supplementary appendix figure 3). By comparison, the number of countries meeting the target for metformin in the public, private and private hospital sectors were 5, 9 and 6 (based on lowest prices) and 4, 6 and 5 (highest prices), respectively.

\section{DISCUSSION}

Of the different countries studied, only the public sector in Brazil, Kyrgyzstan and Pakistan (where insulin is free of charge) and private hospitals in Brazil achieved the GAP target. This study found insulin availability was generally higher in the public sector than the private but still suboptimal and lower than the comparator medicines. Although the causes were not investigated, a prior WHO/HAI study ${ }^{18}$ reported poor medicine availability can be due to a combination of factors including inadequate funding, inability to forecast accurately, lack of incentives for maintaining stocks and inefficient distribution systems. Outlets may regard the need for refrigeration, higher procurement prices and lower demand compared with other medicines as barriers to stocking insulin.

A key issue in accessing insulin is its price. Several price trends were seen in this study. First, governments were paying highly variable prices for the same insulin, such as $\$ 1.45$ (Ethiopia) to $\$ 24.72$ (Shaanxi, China) for isophane human insulin. Even identical products had variable procurement prices. For example, Lantus pens ranged from \$21.56 (Indonesia) to \$106.52 (Hubei, China). Second, analogues were substantially higher priced then human insulin in all sectors. For example, people on insulin paid about $\$ 9.50$ for human insulin, but $\$ 29.39$ and $\$ 43.81$ for analogues in the public and private sectors, respectively. The third trend was that insulins in 
vial form (excluding long-acting and mixed analogues) were cheaper than when in pens and cartridges.

Some LMICs are able to provide insulin for free to their populations, which is an essential step in improving diabetes care. However, in countries where people had to pay for insulin, it was not affordable for those on low wages, requiring 2.2-15.6 days' wages to purchase $10 \mathrm{~mL}$. The comparator medicines were far more affordable at 0.4-2.4 days' wages.

There are different possible areas where individuals and governments could decrease expenditure on insulin. First, the use of analogues is rising and already exceeds human insulin use in upper middle and high-income countries. ${ }^{19}$ This trend should concern all governments facing growing expenditure on medicines for diabetes and other NCDs. On two occasions (2011 and 2017), WHO's Expert Committee on the Selection and Use of Essential Medicines rejected proposals to include analogues in the model list due to their higher price and modest benefit compared with human insulin. ${ }^{20}$ It was again proposed at the 2019 meeting of the Expert Committee, with the outcome unknown at the time of publication of this article. Purchasing human insulin reduces overall expenditure, with the government of Kyrgyzstan potentially saving nearly half a million US dollars annually if only human insulin was purchased. ${ }^{15}$ This trend was seen in an earlier study. ${ }^{15}$ These trends on a global level impact affordability for both governments, when they provide free or subsidised insulin, and people when paying out of pocket. Thus, the provision of human insulin should be promoted.

In late 2018, Gotham and colleagues ${ }^{21}$ conservatively $^{2}$ estimated that manufacturers' selling prices (covering production costs, profit, transport and other costs) should be $\$ 3.29$ to $\$ 4.93$ for biosimilar human insulin and $\$ 5.32$ to $\$ 9.13$ for most analogues. Our study found governments were paying far more, particularly for analogues (but also for human insulins in some countries), showing scope for price reductions while still providing a profit for the manufacturer. This means that, both globally and nationally, pressure is needed on the manufacturers to lower the actual prices at which they sell their insulin.

Efforts are needed to increase competition through the use of lower priced quality-assured biosimilars. ${ }^{22}$ Encouraging biosimilar insulin manufacturers to bid to supply insulin in a country requires procurement agencies knowing who manufactures insulin globally and encouraging them to submit product dossiers for evaluation to the country's medicines regulatory authority. A review of regulatory processes for biosimilar insulins found assessments for these products are more complicated than for chemical medicines, resulting in inconsistent approaches across countries and, in some countries, additional studies being required. ${ }^{23}$ Improvements could be achieved by WHO providing greater guidance combined with building the capacity of national regulators, the inclusion of biosimilar insulin in the WHO prequalification process (WHO have announced they are willing to 


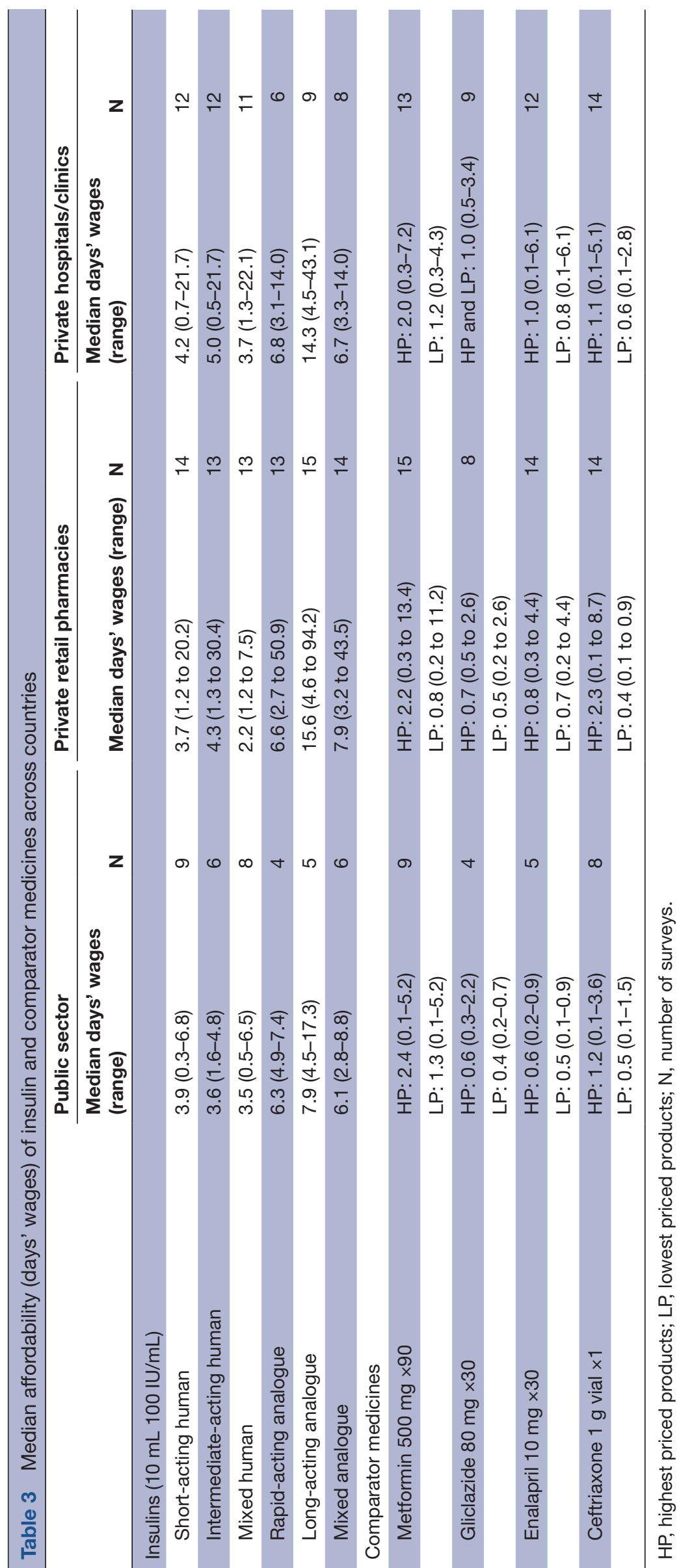

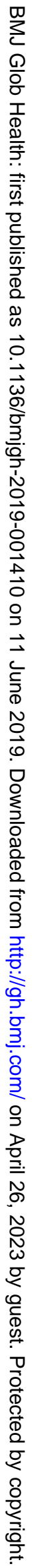




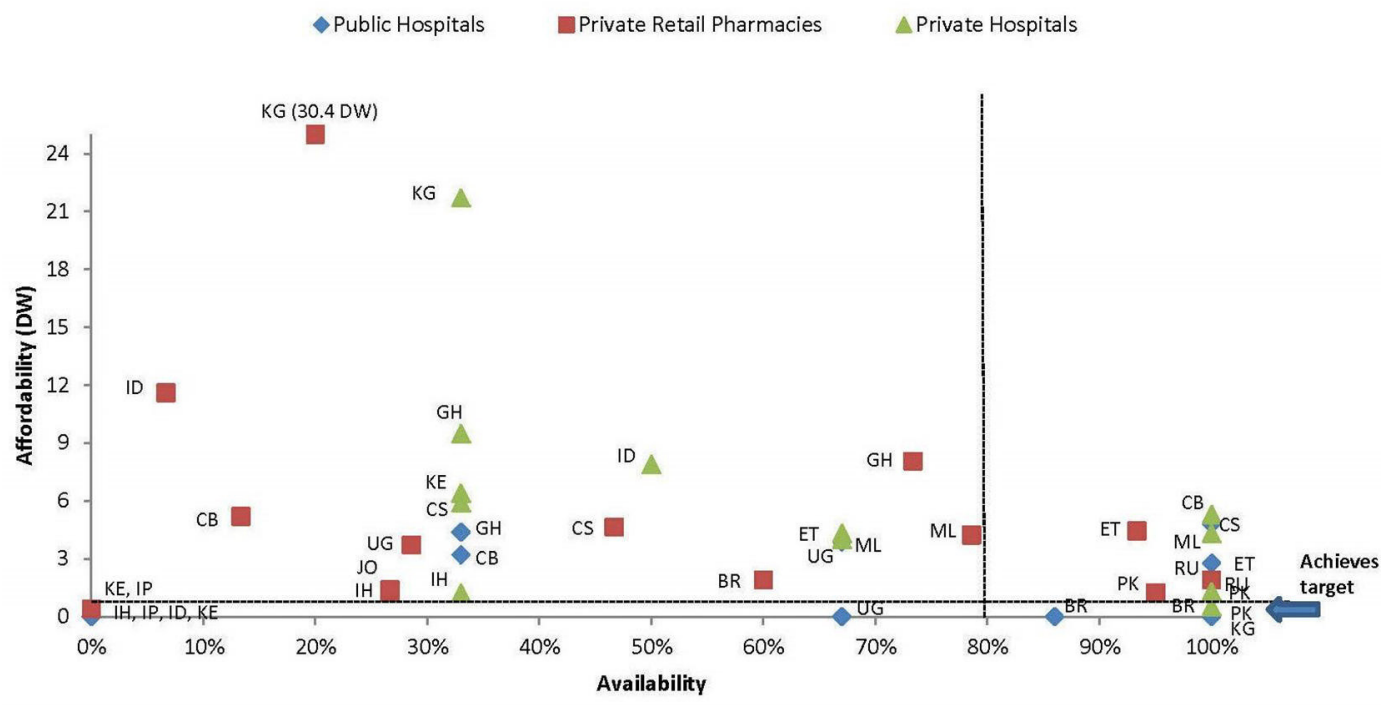

Figure 2 Affordability and availability, isophane (intermediate acting) human insulin, per country and sector. Availability: 0\% in public sector in IH, IP, ID and KE, 0\% in private pharmacies KE and IP and 0\% in private hospitals in IP. BR, Brazil; CB, Hubei, China; CS, Shaanxi, China; DW, days' wages; ET Ethiopia; GH, Ghana; ID, Indonesia; IH, Haryana, India; IP, Madhya Pradesh, India; JO, Jordan; KE, Kenya; KG, Kyrgyzstan; ML, Mali; PK, Pakistan; RU, Kazan, Russia; UG, Uganda.

consider this), ${ }^{24}$ and the harmonisation of evaluations to enable accelerated product approval and market access. The challenge remains that the insulin market is dominated by the three originator companies (Novo Nordisk, Lilly and Sanofi) that have over $90 \%$ market share by volume and value. ${ }^{25} 26$ The remainder is shared by the estimated 10 or so independent biosimilar insulin manufacturers. Discussions with several companies revealed they were manufacturing below maximal capacity that impacts unit prices and their ability to compete with the multinationals. ${ }^{27}$

At a national level, governments paying high prices for insulin need to review their procurement process and eliminate requirements that could negatively impact insulin supplies. In nearly half the surveys, the government procured insulin from only one manufacturer. Consideration should be given to splitting tenders where there are few suppliers but with competitive bids to incentivise staying in the market and help drive prices down over time. ${ }^{28}$ Governments in low-income countries should also assess insulin price discount schemes ${ }^{29}$ as they may (or may not) provide lower prices than can be achieved by other means. Negotiating prices should also be considered. Despite buying relatively small quantities, the United Nations Relief and Works Agency for Palestine Refugees in the Near East achieved significant insulin price reductions through negotiation. Paying on time is also important. ${ }^{30}$

Efforts are also needed to improve the understanding of healthcare professionals and insulin users on the meaning of 'biosimilar' insulin, and the product evaluation process, to build confidence in the use of these products. ${ }^{31}$

There were several limitations to this study: (1) availability data only refer to the day of data collection; however, they reflect the real-life situation faced by insulin users seeking supplies; (2) primary healthcare outlets were mostly excluded as many LMICs do not permit them to dispense insulin, which adds to the access burden; (3) in many countries, a substantial proportion of the population earns less than the lowest paid unskilled government worker hence the affordability analysis may be an underestimate; and (4) other treatment costs, such as glucometers, test strips and syringes, were excluded.

The WHO's former director general stated in 2016 'People with diabetes who depend on life-saving insulin pay the ultimate price when access to affordable insulin is lacking., ${ }^{32}$ Despite being used to treat diabetes for nearly a century, our study shows that insulin prices are high, availability is poor and insulin is not affordable in many contexts. To date, little practical guidance has been provided to countries to help improve insulin availability and affordability. To assist governments and others, the ACCISS Study has developed a toolkit of resources to help address these issues. ${ }^{33}$ However, addressing access to insulin is not enough. With global targets in the Sustainable Development Goals on Universal Health Coverage and NCD mortality, wider health system elements will also need to be addressed, including delivery of care and education. It is hoped that by the centenary of the discovery of insulin (2021), many more countries will be closer to meeting the GAP target and improving the health of those needing insulin.

Acknowledgements The authors are very grateful to the national investigators who collected the data, and the governments and staff in the outlets who so willingly provided the data

Contributors ME, DB and RL designed the study. ME and H-JJ checked and analysed the data. ME drafted the article, and $\mathrm{H}-\mathrm{JJ}, \mathrm{DB}$ and $\mathrm{RL}$ reviewed it.

Funding The Leona M. and Harry B. Helmsley Charitable Trust and Stichting ICF fund the ACCISS Study. 
Disclaimer All references and conclusions are intended for educational and informative purposes and do not constitute an endorsement or recommendation from the Helmsley Charitable Trust or Stichting ICF.The analysis included in this paper is that of the authors alone and does not necessarily reflect the views of the Helmsley Charitable Trust or Stichting ICF.

Competing interests None declared.

Patient consent for publication Not required.

Provenance and peer review Not commissioned; externally peer reviewed.

Data availability statement All data relevant to the study are included in the article or uploaded as supplementary information.

Open access This is an open access article distributed in accordance with the Creative Commons Attribution Non Commercial (CC BY-NC 4.0) license, which permits others to distribute, remix, adapt, build upon this work non-commercially, and license their derivative works on different terms, provided the original work is properly cited, appropriate credit is given, any changes made indicated, and the use is non-commercial. See: http://creativecommons.org/licenses/by-nc/4.0/.

\section{REFERENCES}

1. International Diabetes Federation. International diabetes Federation diabetes atlas. 7th edn. Brussels: International Diabetes Federation, 2017.

2. World Health Organization. Global action Plan for the prevention and control of noncommunicable diseases. Geneva: World Health Organization, 2013: 2013-20.

3. Ewen M, Zweekhorst M, Regeer B, et al. Baseline assessment of who's target for both availability and affordability of essential medicines to treat non-communicable diseases. PLoS One 2017;12:e0171284.

4. Abdraimova A, Beran D. Report on the rapid assessment protocol for insulin access in Kyrgyzstan. London: International Insulin Foundation, 2009

5. Beran D, Atlan-Corea C, Tapia B, et al. Report on the rapid assessment protocol for insulin access in Nicaragua. Managua: International Insulin Foundation and Handicap International, 2007.

6. Beran D, Binh TV, Khue NT, et al. Report on the rapid assessment protocol for insulin access in Vietnam. London: International Insulin Foundation, 2009

7. Beran D, Yudkin JS, de Courten M. Access to care for patients with insulin-requiring diabetes in developing countries: case studies of Mozambique and Zambia. Diabetes Care 2005;28:2136-40.

8. Higuchi M. Costs, availability and affordability of diabetes care in the Philippines. Tokyo: Foundation for Advanced Studies on International Development, 2009.

9. International Insulin Foundation. Final report of the International insulin Foundation on the rapid assessment protocol for insulin access in Mali. London: International Insulin Foundation, 2004.

10. International Insulin Foundation. Report of the International insulin Foundation on the rapid assessment protocol for insulin access in Mozambique. London: International Insulin Foundation, 2004.

11. International Insulin Foundation. Report of the International insulin Foundation on the rapid assessment protocol for insulin access in Zambia. London: International Insulin Foundation, 2004.

12. Beran D, Higuchi M. Delivering diabetes care in the Philippines and Vietnam: policy and Practice Issues. Asia Pac J Public Health 2013;25.
13. Beran D, Abdraimova A, Akkazieva B, et al. Diabetes in Kyrgyzstan: changes between 2002 and 2009. Int J Health Plann Manage 2013;28:e121-37.

14. Health Action International. Life-saving insulin largely unaffordable - a one day snapshot of the price of insulin across 60 countries, 2010. Available: http://haiweb.org/what-we-do/price-availabilityaffordability/collecting-evidence-on-medicine-prices-availability/ a-one-day-snapshot-of-the-price-of-a-medicine/

15. Ewen M, Joose H-J, Ashigbie P, et al. Insulin prices profile. Amsterdam, health action international 2017.

16. Chow CK, Ramasundarahettige $\mathrm{C}, \mathrm{Hu}$ W, et al. Availability and affordability of essential medicines for diabetes across highincome, middle-income, and low-income countries: a prospective epidemiological study. Lancet Diabetes Endocrinol 2018;6:798-808.

17. World Health Organization/Health Action International. Measuring medicine prices, availability, affordability and price components. 2nd edn. Geneva, 2008.

18. Cameron A, Ewen M, Ross-Degnan D, et al. Medicine prices, availability, and affordability in 36 developing and middle-income countries: a secondary analysis. The Lancet 2009;373:240-9.

19. Beran D, Ewen M, Laing R. Constraints and challenges in access to insulin: a global perspective. Lancet Diabetes Endocrinol 2016;4:275-85.

20. WHO. Executive summary. The selection and use of essential medicines 2017. Report of the 21st who expert Committee on the selection and use of essential medicines.. Available: http://www. who.int/medicines/publications/essentialmedicines/EML 2017 ExecutiveSummary.pdf [Accessed 9 Aug 2018].

21. Gotham D, Barber MJ, Hill A. Production costs and potential prices for biosimilars of human insulin and insulin analogues. BMJ Glob Health 2018;3:e000850.

22. McCall C. Biosimilars for insulin: a cost-saving alternative? The Lancet 2018;392:463-4.

23. Lapteva M, Singhal M, Kalia Y. Biosimilar insulin regulatory profile. Amsterdam, health action international 2017.

24. Who to begin pilot prequalification of biosimilars for cancer treatment who to begin pilot prequalification of biosimilars for cancer treatment. Available: http://www.who.int/mediacentre/news/ releases/2017/pilot-prequalification-biosimilars/en/ [Accessed 20 Feb 2018].

25. Wirtz V. Insulin market profile. Amsterdam, health action international 2017.

26. Schultz K. The global diabetes care market. Bagsværd: Novo Nordisk, 2011.

27. Perrin C, Ewen M, Beran D. The role of biosimilar manufacturers in improving access to insulin globally. Lancet Diabetes Endocrinol 2017;5.

28. van Valen $M$, Jamieson $D$, Parvin $L$, et al. Dispelling myths about drug procurement policy. The Lancet global yhealth. The Lancet Global Health 2018;6:e609-10.

29. Nordisk N. Access to insulin commitment. Available: https://www. novonordisk.com/sustainable-business/performance-on-tbl/accessto-care/our-access-to-insulin-commitment.html [Accessed 9 Aug 2018]

30. WHO. Operational principles for good pharmaceutical procurement. WHO/EDM/PAR/99.5. Available: http://www.who.int/3by5/en/whoedm-par-99-5.pdf [Accessed 8 Aug 2018].

31. Wilkins AR, Venkat MV, Brown AS, et al. Patient perspectives on Biosimilar insulin. J Diabetes Sci Technol 2014;8:23-5.

32. WHO. Global report on diabetes. Geneva: World Health Organization, 2016.

33. ACCISS Study. Access to insulin toolkit. Available: http:// accisstoolkit.haiweb.org 